\title{
THE PRE - MAIN SEQUENCE
}

\author{
FRANCESCA D'ANTONA \\ Osservatorio Astronomico di Roma, Monte Porzio, Italy
}

ABSTRACT The Pre-Main-Sequence (pre-MS) is defined as the phase following the protostellar stage and ending with the ignition of hydrogen in the stellar core. Models in hydrostatic and thermal equilibrium such as typical "Hayashi track" models are basically adequate to describe the internal structure of pre-MS stars, although for a fraction of pre-MS objects there can be phases of accretion also at late stages, which may influence the surface abundances of light elements.

The interior evolution of pre-MS structures is focused in the two main stages of Deuterium and Lithium burning for the low mass $(M \leq$ $\left.1.5 M_{\odot}\right)$ stars. The location in the HR diagram of theoretical tracks is still subject to large uncertainty, even more apparent today that new opacities and treatment of turbulent convection are available, and the results can be internally compared. Uncertainties amount to a factor $\sim$ two for mass and age determination of individual objects, and affect the absolute location for both the $\mathrm{D}$-burning and the $\mathrm{Li}$-burning regions. Qualitative constraints, such as that there can not be Li-depletion at $L \geq L_{\odot}$, remain valid. A bundances of ${ }^{7} L i$ at the surface of pre-MS stars, if measured at a stage which can definitely be considered previous to the possible occurrence of nuclear burning, and, consequently, previous to other depletion mechanism which may occur later on, provide constraints on galactic evolution of this element of cosmological interest.

\section{DEEINITION: THE PRE-MS AND THE PROTOSTELLAR PHASES}

The first phases of stellar evolution may bear important consequences for the understanding of normal stars. Great progress has been made in the latest years, both theoretically and observationally, and this review will try to focus on a few aspects, more than try to give a complete overview of the work done or in progress.

As the same definition of pre-MS stage is subject to some ambiguity, I will define as pre-MS for low masses $\left(M \leq 1.5 M_{\odot}\right)$ the phase starting in the vicinity of deuterium burning in fully convective structures, and ending with the onset of hydrogen burning in the stellar core (MS).

The proto-stellar phases begin with the free-fall collapse of interstellar clouds. This highly non-homologous collapse (Larson, 1969), with the high density central regions collapsing first, soon leads to a configuration in which a central hydrostatic core gravitationally accretes the remaining diffuse matter 


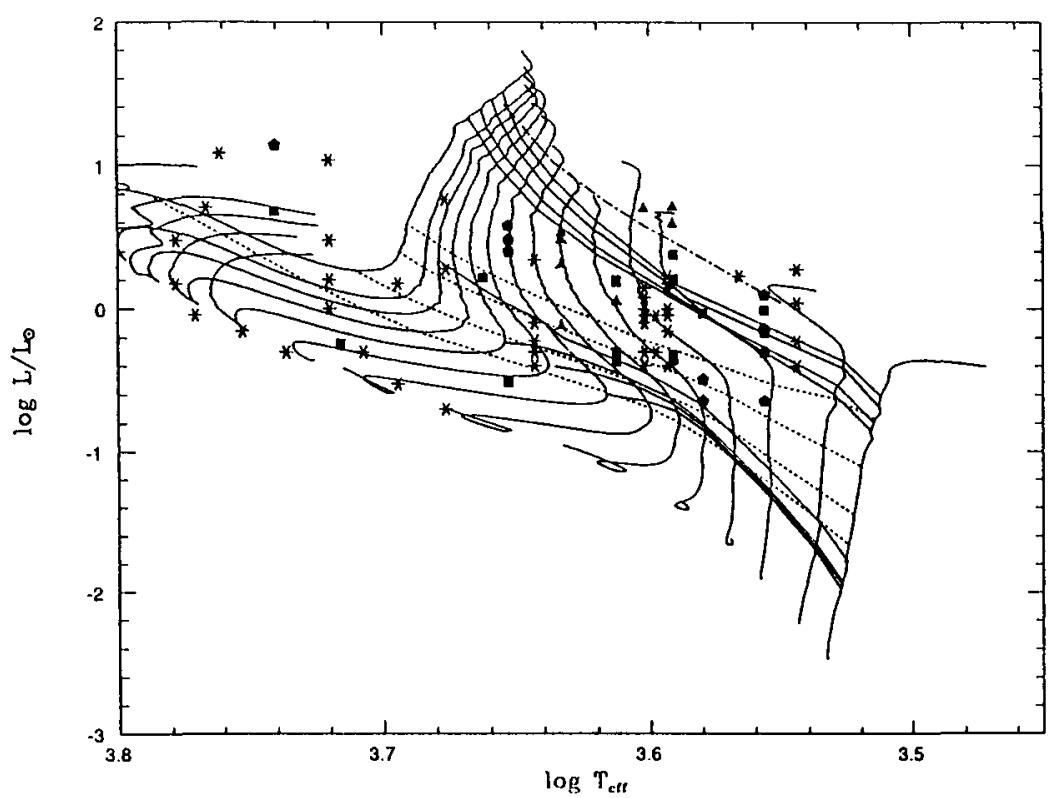

FIGURE I Representative evolutionary tracks of $1.5,1.3,1.2,1.1,1.0$, $0.9,0.8,0.7,0.6,0.5,0.4,0.3,0.2,0.15 M_{\odot}$, on which we show: $i$ : the four upper continuous lines are the locus at which the nuclear luminosity due to D-burning reaches $20 \%, 60 \%$, then goes down to $60 \%$ and $20 \%$; the uppermost dash-dotted line shows how the region of $L_{n u c}=20 \%$ for D-burning is shifted in models having different opacities; $i$ : dotted lines: isochrones of $1,2,5 \times 10^{6}$ and $10^{7} \mathrm{yr} ; i i i$ : the four lowest continuous lines represent, from top to bottom, beginning of $\mathrm{Li}$-burning and lines at which the initial abundance is reduced by $.5,1$ and 2 dex respectively; $i v$. observational data collected by Magazzù et al.(1992) and Basri et al., 1992.

in the outer regions. The protostar is obscured by the dust surrounding the parent cloud, and it will emerge in visual light only when the main accretion phase is ended (Stahler, 1983). During the protostar phase, probably during the bipolar molecular outflow phase, the object gets rid of most of the Angular Momentum (AM) of the parent cloud. Consideration of AM evolution (see Pringle, 1989, Bodenheimer, 1991) should be a fundamental ingredient in the study of protostellar evolution. When low-mass pre-MS objects appear, at least those identified with T-Tauri's are already slowly rotating (Bouvier, 1991).

The onset of a strong stellar wind ends the accretion phase, disperses the dust, and lets the pre-MS star emerge. It is to be explained why the stellar wind begins, what are the physical parameters which determine the Initial Mass Function of the stars, and how the very low mass stars are formed, but this approach provides at least a phenomenological description. According to Stahler (1983), the pre-MS star appears in the HR diagram at the intersection between the Hayashi line of fully convective hydrostatic stellar models and the massradius relation of the hydrostatic core. The "stellar birthline" depends on the stellar mass mainly (Palla and Stahler, 1992). D-burning is the key process 
occurring in the protostar and determining its final mass-radius relation (Stahler 1988). In stars of $M \leq 2 M_{\odot}, D$ is ignited in the core and forces the star to become fully convective (Stahler, Shu and Taam, 1980). At larger masses, a more complicated behaviour sets in, due to the onset of a radiative barrier and the subsequent ignition of $D$ in a shell (Palla and Stahler, 1990,1991). In the absence of $\mathrm{D}$-burning, the accretion timescale for $M \gtrsim 3 M_{\odot}$ is longer than the simple Kelvin Helmoltz timescale $\left(t_{K H}\right)$, and masses would directly appear on the MS. Introduction of D-burning then explains why pre-MS stars of these masses exist (see for a review on the subject Palla, 1991).

For masses $M \leq 1.5 M_{\odot}$, the location of the birthline will depend on the details of the protostar evolution (e.g. boundary conditions and accretion rate), but, qualitatively, if the protostar phase is thermostated by D-burning, the preMS stars upper envelope in the HR diagram should coincide with the locus of D-burning in hydrostatic stellar models. Palla and Parigi (1992, in these proceedings) show that a $1 M_{\odot}$ pre-MS star appears below the locus of D-burning, evolves to it by increasing $L$, and then follows the Hayashi line evolution. The thermal disequilibrium induced in the core during the accretion should soon be over: the star will first adjust on a hydrostatic equilibrium timescale, and then completely relax within a Kelvin-Helmoltz time, which is of the order of the former accretion timescale (see, e. g., Mercer Smith et al., 1984). From this moment on, the stellar evolution occurs on the thermal timescale, apart from the momentary stop due to $\mathrm{D}$-burning. When a radiative core appears and convection in the envelope progressively recedes, the star begins evolving towards the main sequence. The possible burning of other light elements $\left({ }^{6} \mathrm{Li},{ }^{7} \mathrm{Li},{ }^{9} \mathrm{Be}\right)$ is to be considered during this phase. Finally, hydrogen is ignited in the core and the pre-MS phase can be considered concluded.

In this review I concentrate on the following problems: $i$ : the D-burning phase; $i i$ : the possible influence of residual accretion on the pre-MS evolution; iii: the uncertainty of stellar modeling and the location of the tracks in the HR diagram; $i v$ : the burning of light elements and the new data on the Lithium in pre-MS objects.

All the following discussion will be based on the results of "standard" stellar modeling (one-dimentional non rotating stars without magnetic fields, mass loss or accretion and without rotation). It is then mandatory to add a few comments regarding rotation.

Although at least those pre-MS low mass stars identified with TTauri's are slowly rotating, and the pre-MS begins when the protostellar phases have already acted to eliminate most of the Angular Momentum (AM) of the forming cloud (see, e.g. Bodenheimer, 1991), we should not, in principle, ignore the evolution of AM during the pre-MS stage. Inclusion of an initial AM compatible with the observed rotations of TTauri's does not, in itself, imply drastic changes in the structure of the star or in its observable properties, as shown, for instance, by the "1.5"-dimensional models by Pinsonneault et al., 1990, which account for rotation adopting the approach by Endal and Sofia (1981). Most important, and subject to uncertainty, is the evolution of AM during the pre-MS phase, which, in principle, has to take into account both loss of AM at the stellar surface, due, probably, to magnetic winds, the possible advection of AM from the circumstellar disk, the establishing of gradients of rotational velocity through the structure when the radiative core appears, and its consequent transport and 
redistribution. The final MS stellar rotation distribution is dictated both by the initial rotation (as solid body, if the star is initially fully convective) and by the parameters which regulate loss, advection and redistribution of AM. The models by Pinsonneault et al. (1990) represent by now an interesting step in the exploration of the parameter space.

Further, many mechanisms which alter the surface composition of MS stars depend on rotation (e.g. meridional circulation) and also it is probable that chemical mixing is associated with the redistribution of AM: this may be an additional cause of depletion of light elements at the surface of stars (see, for a review, Demarque, 1991) and adds to the importance of exploring the evolution of rotating models. For what concerns this review, however, I notice that the timescale on which the effects of mixing associated with the AM transport takes place is of several $\times 10^{7} \mathrm{yr}$, so that the AM distribution achieved within the star during pre-MS evolution might have long-term consequences during the MS lifetime, and not, mainly, in the pre-MS stage itself. For instance, the models by Pinsonneault et al., 1991, show that the effect of rotation on Lithium depletion is not dramatic till an age of $30 \mathrm{Myr}$, while it grows up to a factor $0.3-0.6 \mathrm{dex}$ (depending on $T_{\text {eft }}$ ) already at $70 \mathrm{Myr}$.

My conclusion is then that we can outline the broad lines of surface evolution of light elements in pre-MS without accounting for rotation, but rotation may be of great relevance for the interpretation of abundances and rotation evolution during the MS.

\section{THE D-BURNING PHASE}

As a general reference for our discussion I show in figure I the HR diagram with representative tracks of masses from 1.5 to $0.15 M_{\odot}$, with population I chemical inputs, and "updated" physics. The general framework in which these tracks were computed is in part described in next section and will be presented in a forthcoming paper by D'Antona and Mazzitelli (1992). The loci of D- and Liburning are shown, as well as a few isochrones The points represent T-Tauri's in several star forming regions.

The four curves at the top of the tracks represent the main locus of Dburning. In this diagram several pre-MS appear above this region. This can be attributed in part to the influence of the disk on the apparent location of TTauri (Kenyon and Hartmann, 1990, see section 3), in part to the fact that the location of the tracks in the HR-diagram is very model-dependent (section 4), as shown by the dot-dashed line. The fact that actually TTauri's appear more or less at the "birthline" is suggestive, but it is not a definite proof that Stahler's scenario is correct. Actually, as we see in figure II, where I plot the luminosity versus time along the tracks, the D-burning stage is the first one during which the star slows down its evolution. It lasts, in fact, from $\lesssim 10^{5} \mathrm{yr}$ for masses $\sim 1 M_{\odot}$ to $\sim 10^{6} \mathrm{yr}$ for $\mathrm{M} \leq 0.3 M_{\odot}$ or smaller: even if the pre-MS stars begin their life at larger luminosity, their luminosity function would be such that it is more probable to observe them only when they reach the D-burning line. Recently, Zinnecker et al. (1992), on the basis of models by Mazzitelli (1990, unpublished), have discussed how the D-burning locus could even be observed as a "peak" in the luminosity function of pre-MS stars. 


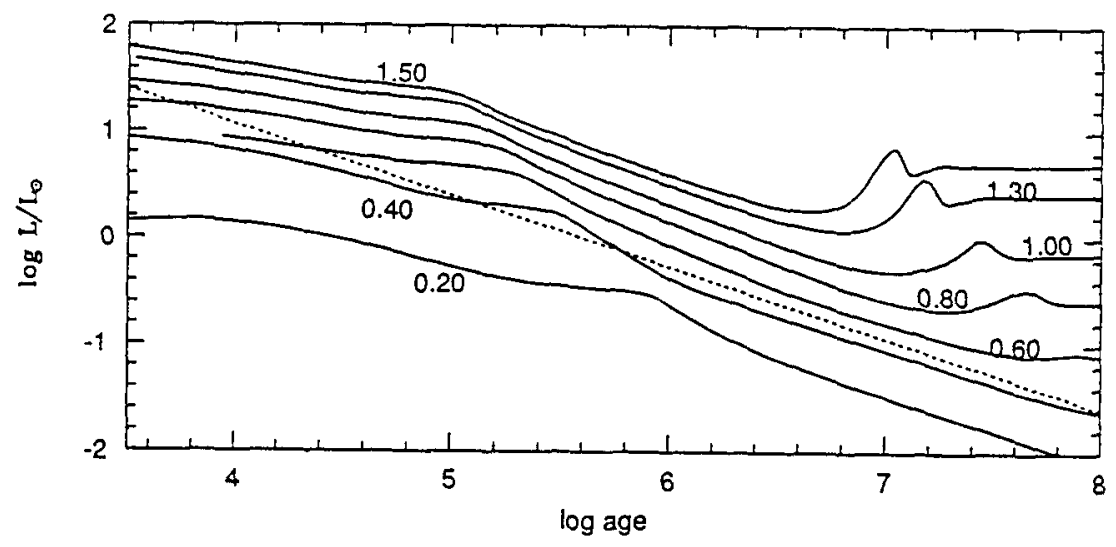

FIGURE II Luminosity evolution versus log age (in years) for models from $1.5 M_{\odot}$ to $0.2 M_{\odot}$. The first flattening of the curves is due to the $\mathrm{D}-$ burning (taken to be $X_{D}=10^{-5}$ in mass fraction) and the second one is due to reaching of the main sequence. The diagonal line represents a curve of slope $-2 / 3$, which is followed by stars simply contracting at fixed $T_{\text {eff }}$. The stop due to D-burning does not alter substantially this type of evolution for most stellar masses.

The exact duration depends on the abundance of $D$ in the star forming clouds. Computations in figure II refer to $X_{D}=2 \times 10^{-5}$, where $X_{D}$ is the Deuterium mass fraction, or a number fraction $[D / H] \sim 10^{-5}$ (York and Rogerson, 1976, Boesgaard and Steigman, 1985) but values as low as $[D / H] \sim 5 \times 10^{-6}$ (Vidal Majar et al. 1983), or as large as $2.5 \times 10^{-5}$ (Bruston et al., 1981), as in the solar nebula, are quoted in the literature. The diagonal straight line is the typical $L \sim t^{-2 / 3}$ evolution expected on the basis of simple contraction along a Hayashi line at fixed $T_{\text {eff }}:$ D-burning does not alter substantially this evolution.

For masses $M \leq 0.1 M_{\odot}$, the D-burning lasts for a relatively long time (1$2 \times 10^{6} \mathrm{yr}$, see e.g. D'Antona and Mazzitelli 1985, Pozio, 1990), and its locus becomes almost vertical, at a $T_{\text {eff }}(\sim 3000 \mathrm{~K})$ (this value of $T_{\text {eff }}$ is highly uncertain, as it depends on the opacities and other assumptions of the models). Thus, coeval pre-MS stars of low mass must appear at this $T_{\text {eff }}$, with $L$ decreasing with decreasing mass, and it is, in principle, possible to try to derive information on the Initial Mass Function (IMF) of young clouds associations from the luminosity function of these objects. This has been attempted for the $\rho$ Oph cloud (Rieke et al., 1989, Barsony et al., 1989, Rieke et al., 1991). In view of the difficulty of observational discovery of Brown Dwarfs (if they exist, see, e.g. Stevenson, 1991), it is very important to try to find them when they are young and more luminous, in spite of the difficulties which interpretation of these results may bear (see, e.g., Zinnecker et al., 1991).

Grossman and Graboske (1973), have shown that $D$ is not ignited in masses $M \lesssim 0.01 M_{\odot}$. Pozio $(1990,1991)$ confirmed this result and found that there exists a small mass range (identified with $M \sim 0.015 M_{\odot}$ ) at which the conditions for $D$-burning $\left(T_{\text {center }} \sim 1.1 . \times 10^{6} \mathrm{~K}, \rho_{\text {center }} \sim 6 \mathrm{~g} \mathrm{~cm}^{-3}\right)$ are reached at a very low luminosity $\left(\sim 10^{-3.5} L_{\odot}\right)$, so that the phase lasts much longer than usual, 
$\sim 10^{8} \mathrm{yr}$, mimicking a true $D$-burning main sequence. We might be able to find a signature of this event when looking for very red low luminosity $\mathrm{BD}$ candidates.

\section{RESIDUAL MASS ACCRETION AND ITS POSSIBLE INFLUENCE}

Recent observations have shown that the Classical T Tauri (CTTs), stars characterized by strong emission lines indicating both accretion and mass loss (e.g. Bertout, 1989), veiling of the lines and UV and IR excesses over a continuum of spectral type from $K$ to $M$, are not the only pre-MS objects: Naked (or Weak line) T Tauri's (WTTS) have been discovered as soft X-ray sources, and afterwards identified with low mass pre-MS stars (Walter et al., 1988).

The properties of CTTS are consistent with a model of a star plus a keplerian accretion disk and a boundary layer, as suggested already by Lynden Bell and Pringle (1974). Recent modeling (Adams et al., 1987, Kenyon and Hartmann, 1987, Bertout et al., 1988) lead to infer mass accretion rates in the range from $\sim 3 \times 10^{-9}$ to $4 \times 10^{-7} M_{\odot} /$ yr (Basri and Bertout, 1989). The WTTS, among which $\sim 30 \%$ present small IR excesses in the $K$-band $(2.2 \mu)$, can be modeled again with a star plus a disk, but with no boundary layer and a central cavity (possible site where planets have formed?) (Montmerle and André, 1989). Walter (1987) and Strom et al. (1989b) show that both CTTS and WTTS occupy the same region of the HR diagram which, interpreted with normal hydrostatic contraction models, range from ages of $\sim 3 \times 10^{5}$ to $\sim 3 \times 10^{6} \mathrm{yr}$. This probably indicates that disk evolution is decoupled from the evolution of the central star and that the disk may disappear either early or at a very late stage.

How much the evolution can be altered by mass accretion? The $D$-burning luminosity which would be provided by burning of the accreted $D$ is generally negligible, being $L_{D} \sim 15 L_{\odot} \times\left(\dot{M} /\left(10^{-5} M_{\odot} / y r\right)\right)-$-e.g. Stahler, 1988. This could have an influence either for accretion rates much larger than observed, or at very low luminosity. Accretion itself may alter the evolution only if we have: $M / \dot{M}_{\text {crit }} \sim t_{K H}$. At large luminosity, where $t_{K H} \sim 10^{5} \mathrm{yr}$, this constraint would require too large mass accretion rates. At $L \sim L_{\odot}$, we have $t_{K H} \sim 2 \times 10^{6} \mathrm{yr}$, for which $\dot{M}_{\text {crit }} \sim 5 \times 10^{-7} M \odot / \mathrm{yr}$, which is at the upper boundary of what observed in CTTS: in this case the evolution can be probably slightly altered, but it is clear that such a $\dot{M}$ can not be mantained for a very long time, as low mass stars exist. We conclude that accretion can be important for the evolution of a fraction of the CTTS (an even smaller fraction of all pre-MS stars), only for short periods of time.

The "post-T Tauri problem" (lack of TTs of age $\sim 10^{7} \mathrm{yr}$ in the Taurus Auriga complex, Herbig et al., 1986), stressed also by the negative results of the proper motion survey by Hartmann et al. (1989), is probably to be interpreted in terms of existence of separated loci of star formation in Taurus, clustered at different ages (Walter, 1987).

\section{THE ABSOLUTE LOCATION OF TRACKS IN THE HR DIAGRAM}

I conclude that the evolution of pre-MS stars is well described by hydrostatic stellar models, which are mainly dominated by the stellar contraction. Of course the absolute location of these tracks in the HR diagram, and thus both the mass 
calibration of the MS, the locus of D-burning, and, as we shall see, the locus of Lithium burning, are subject to several sources of uncertainties. Mazzitelli (1989) has examined four main aspects of the input physics of stellar models which influence the HR diagram location of the tracks, namely: $i$ : the convection model; $i i$ : opacity; iii: thermodynamics; $i v$ : rotation, and I refer to that paper for the general discussion. Recently we have acquired several improvements in the input physics, which eventually should lead us to a better understanding of the HR diagram location of the tracks.

\subsection{The convection model}

Turbulent convection in stars is generally treated according to the Mixing Length Theory (MLT, Böhm Vitense, 1958), in which the free parameter $\alpha$ (ratio of the mixing length $l$ to the pressure scale height $H_{p}$ ) is tuned so as to fit the solar radius. Canuto and Mazzitelli (1992) have explicitly shown that this procedure, when applied to stars different from the Sun, is absolutely unsafe and likely to introduce systematic hidden errors. This is not a very new argument, but it is generally not considered at all, in the absence of a better description of turbulence.

We are presently testing the model which Canuto and Mazzitelli (1990, hereinafter CM) have proposed as an alternative to the MLT. It accounts more precisely for the nature of turbulent convection, by two main improvements: first, the entire distribution of energies of all eddies is taken into account, while the MLT is a "one-eddy" description; second, the mixing length is chosen to be the distance $(z)$ from the point in which the flux is to be computed to the top of the convective region. Therefore, there are no free adjustable parameters in the models, although small adjustments can be done in the choices of a a few input values for the model, in a range allowed by experimental determinations. After the successful application of the new model to the Sun (CM), we have recently shown that it is able to reasonably fit the HR diagram of an old open cluster like M67 (D'Antona et al., 1992): in particular, the model can fit the giants and clump stars location. Although it is clear that much work is still to be done before we can claim to have a better convection model, and problems exist, in particular for cool, expanded structures, for defining properly the boundary conditions and thus for using an appropriate value of $z$, it is necessary to continue the exploration, for example by applying it to the treatment of pre-MS structures.

It is worth to enphasize one of the characteristic of the new treatment of convection: if we compare the new tracks with tracks computed with the MLT we immediately see that the ratio $\alpha=l / H_{p}$ which fits the main sequence -in particular, which fits the solar $T_{\text {eff }}$ - does not provide giants having the same $T_{\text {eff }}$ as the $C M$ model. Although this does not guarantee at all that the CM model provides a definitive description of giant structures -a compressible turbulence theory is needed for that-, nevertheless it provides another strong warning against extending the $\alpha$ value calibrated on the Sun to stars other than the Sun itself! Figure III shows in fact three different tracks for the solar model. The continuous line gives the evolution of a model which should in principle be the most updated possible for choice of opacities and equation of state. It ${ }^{-1}$ so includes CM convection model. The solar $T_{\text {eft }}$ at an age of $4.6 \times 10^{9}$ yr is 


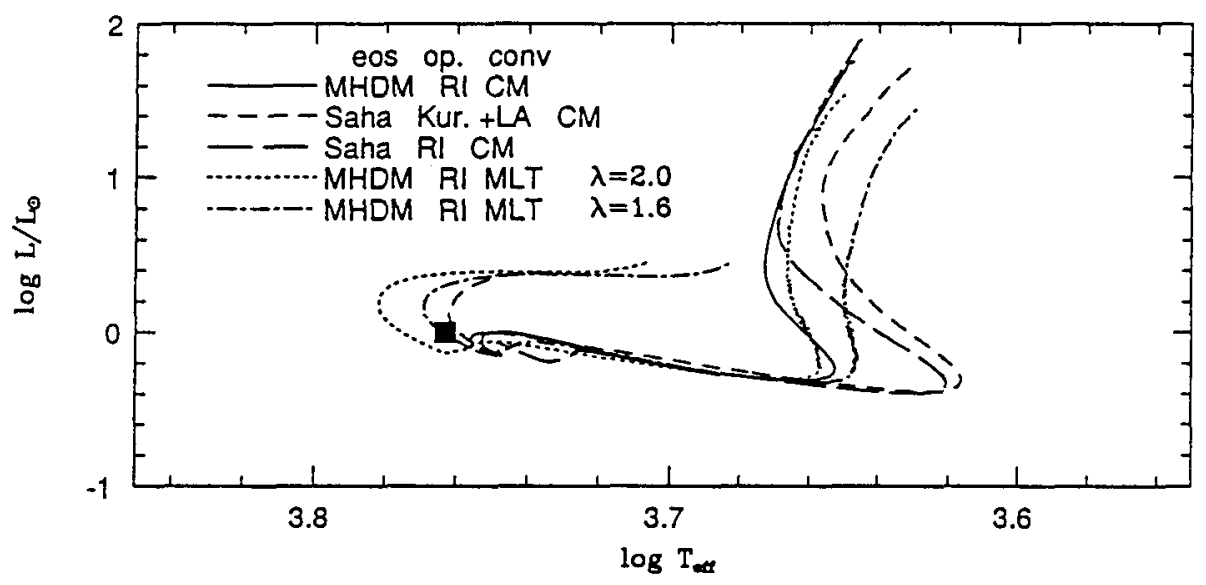

FIGURE III Five different pre-MS tracks are shown for the solar model, identified by adopted EOS, opacities (RI $\equiv$ Rogers and Iglesias, 1992; Kur. $\equiv$ Kurucz, 1991; LA $\equiv$ Los Alamos) and convection model (CM or MLT).

$\sim 50 \mathrm{~K}$ smaller than the real solar $T_{\text {eff }}$ (Canuto and Mazzitelli 1992). Two other evolutionary tracks are shown, having the same physical inputs and numerics, but adopting the MLT for the computation of superadiabatic convection. In order to fit the solar position (which, in this case, can be fitted better than within $50 \mathrm{~K}$ ), we need $\alpha=1.6$. But we see that, in order to reproduce the giant pre-MS location of the CM model we have to fix $\alpha \sim 2$.

As a conclusion, a better description of turbulence seems to give hotter giants in pre-MS than those obtained by adopting the same $\alpha$ which fits the Sun. The opacity uncertainties are still too large to hope to find a test of this feature, by now.

Although convective efficiency does not alter very much the burning of light elements (mainly Lithium), it is a well known result of computations that tracks with a larger $\alpha$ present a somewhat larger depletion (e.g. Deliyannis et al., 1990). As CM models in the giant phase correspond to MLT models with larger $\alpha$, they also produce a slightly larger $\mathrm{Li}$ - depletion than MLT models employing the standard $\alpha$ calibrated on the Sun.

\subsection{The equation of state}

The importance of employing a good Equation of State (EOS) for the solar and stellar models has been discussed in this colloquium by Däppen (1992). In what concerns the pre-MS evolution, we simply add that use of a non-ideal gas EOS narrows the partial ionization region and then lowers the values of the adiabatic gradient there (e.g. Graboske et al., 1975, Magni and Mazzitelli (1979), Saumon, 1990). The "standard" $1 M_{\odot}$ track shown in figure III makes use of the Mihalas et al.(1988) EOS (MHDM) for the region in which it is available (densities $\rho \leq 0.01 \mathrm{~g}$ $\left.\mathrm{cm}^{-3}\right)$. It is compared with a corresponding track employing a simple Saha type EOS in the same region. We see that the MHDM EOS produces larger $T_{\text {eff }}$ 's in the region where the track departs from the Hayashi track to begin approaching 


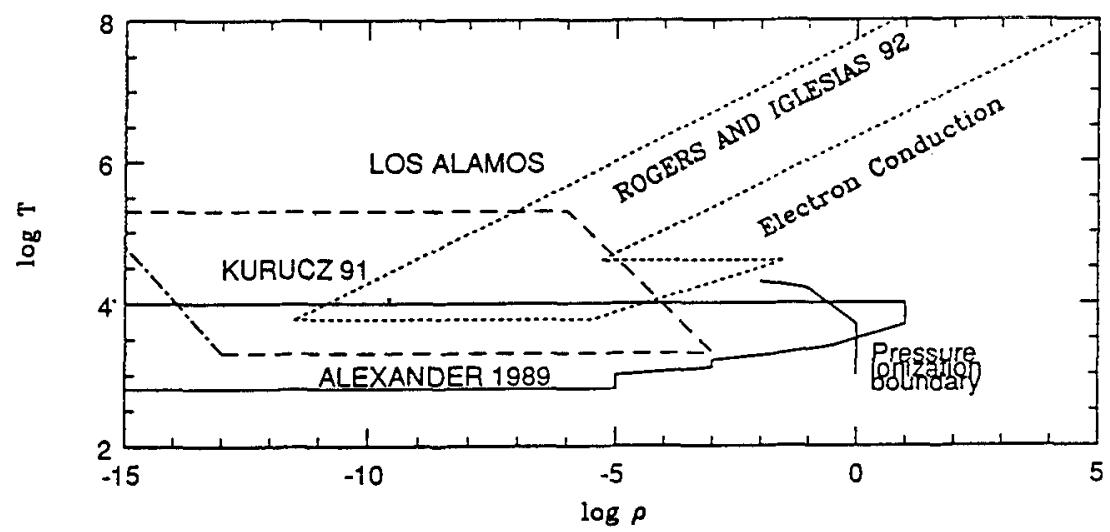

FIGURE IV Coverage of the density - temperature plane by means of available opacity tabulations

the MS. In practice, the "nose" of the track is less pronounced. Apart from the different location of masses in the HR diagram, this feature has a small but noticeable influence on the burning of Lithium: the conditions at the base of the convective envelope in the models employing the MHDM EOS, are favourable to more efficient burning.

\subsection{Opacities}

In spite of the great recent improvements of these latest years, this remains the softest spot of pre-MS evolution. I show in figure IV the $\rho-T$ plane where the different regions covered by recent available opacity computations are indicated. Previous to 1991, one choice was obligatory: Los Alamos (Huebner et al., 1977) tables. These had to be supplemented by low temperature opacities (generally from Cox and Tabor, 1976). An important effort was done by Alexander to include molecules and grains in the computations. Actually, Alexander et al. (1989) complete set of low temperature opacities with updated treatment also of water vapour is, till today, the most extended available table in density, although use of these tables must take into account that the opacities at the largest densities are not to be considered, as they are past the pressure ionization boundary (figure IV). Alexander's opacities are given only for solar composition, and this prevents many applications, although it is largely sufficient for the preMS.

Kurucz (1991) presented opacities at low temperature for a large number of chemical compositions. These are not extended enough to completely cover the low mass external layers regions, but they are adequate for the pre-MS. Rogers and Iglesias (1992) opacities (hereinafter RI) for $T \geq 6000 \mathrm{~K}$ cover most of the interesting regions of the $\rho-T$ plane, although they should be extended to larger densities, if one wants to use them for low mass stars. Use of RI opacities is recommended on the basis of the improved treatment of the physics they adopt (see for a discussion Däppen, 1992, in these proceedings), and although they must be supplemented with the Los Alamos opacities in the regions where they 


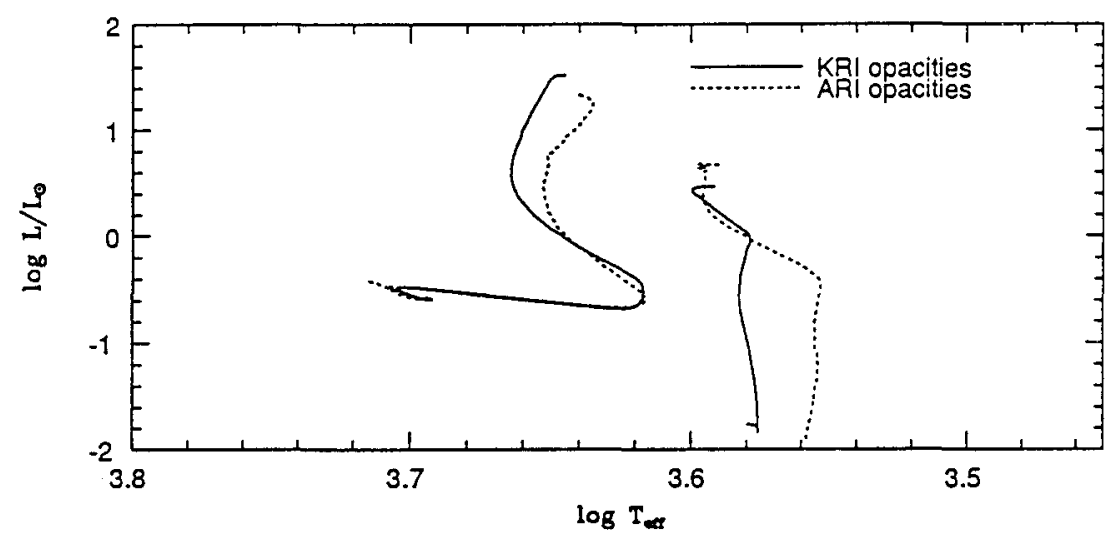

FIGURE V HR diagram for 0.8 and $0.3 M_{\odot}$ tracks for different opacity inputs, as explained in the text.

are still not available. At large densities, in the regions of full ionization, any available radiative opacity must be complemented with the electron conduction, but this does not interest pre-MS evolution.

The influence of the latest available choices (ARI: Alexander at $T \leq 10000 \mathrm{~K}$, plus RI; and KRI: Kurucz at $T \leq 6000 \mathrm{~K}$, plus RI) for pre-MS evolution is shown in figure $\mathrm{V}$, where I compare the different tracks obtained for 0.8 and $0.3 M_{\odot}$ (CM convection model is adopted). The uncertainty in the $T_{\text {eff }}$ determination is quite large especially for $0.3 M_{\odot}$, where it becomes $\sim 0.03 \mathrm{dex}$.

\section{THE BURNING OF LIGHT ELEMENTS}

Depletion of light elements other than $D$ in the pre-MS phase interests mainly burning of ${ }^{6} \mathrm{Li},{ }^{7} \mathrm{Li}$ and ${ }^{9} \mathrm{Be}$, which occur as soon as the internal temperatures reach respectively $\sim 2, \sim 2.5$ and $\sim 3.5 \times 10^{6} \mathrm{~K}$. These temperatures are only indicative: in very low mass stars, and in advanced phases in which the bottom of the convective layer is situated at larger densities, the temperatures of burning are smaller. A $0.08 M_{\odot}$ star ignites ${ }^{7} L i$ at $T_{c} \sim 1.9 \times 10^{6} \mathrm{~K}$ and $\rho_{c} \sim 26 \mathrm{~g} \mathrm{~cm}^{-3}$ (Pozio, 1990).

Extensive works on standard pre-MS depletion of light elements, and in particular of Lithium, are available in the literature, starting from Bodenheimer (1965), to D'Antona and Mazzitelli (1984), and up to Vandenberg and Poll (1989) and Swenson et al., 1989). Depletion of ${ }^{6} \mathrm{Li}$ has been considered in detail by Proffitt and Michaud, 1989, and Deliyannis et al., 1990 (for metal poor stars). Burning of ${ }^{9} \mathrm{Be}$ is considered by Deliyannis and Pinsonneault, 1990 and Ryan et al., 1992. All these elements have a cosmological interest, being synthetized during the Big-Bang (see, e.g. Boesgaard and Steigman, 1985), and it is important to compare detailed models of depletion with the observations, in order, for instance, to discriminate between different models, or to test different formation scenarios.

The galactic evolution of ${ }^{7} L i$, in particular, is a fundamental topic which 
still is subject to large uncertainties (for a general discussion see, e.g., Mathews et al., 1990, D'Antona, 1991, D'Antona and Matteucci, 1992). The fundamental problem in depicting galactic evolution is that it is not clear how much the observed abundances at the surface of population II stars reflect the primordial abundance. Depletion mechanisms for the light elements can be divided into two broad categories: "fast" mechanisms, which occurr on a short timescale: these are mainly burning in pre-MS $\left(\tau \sim 10^{7} \mathrm{yr}\right)$ and dilution as soon as convection deepens into the star evolving as a giant; "slow" mechanisms, which cause depletion on longer timescales, such as diffusion (Proffitt et al., 1990, Deliyannis and Demarque, 1991), mixing associated with transport of angular momentum (Pinsonneault et al., 1990) or by waves (Garcia-Lopez and Spruit 1991, Schatzman, 1991), having $\tau \sim 10^{8}-10^{9} \mathrm{yr}$.

$I$ list the few, probably firm results on ${ }^{7} L i$-pre-MS burning:

1. ${ }^{7} \mathrm{Li}$ is not touched either in stars in which the radiative core appears so early during the evolution that temperatures necessary for burning are never reached at the convective bottom (conservatively, for $M \gtrsim 1.4 M_{\odot}$ ), or in objects which are so small in mass that degeneracy prevents increase of central temperature beyond burning conditions. Pozio (1991) finds that this lower limit is $M \sim 0.06 M_{\odot}$. Rebolo et al.(1992) propose to use the absence/presence of the Lithium line in candidate Brown dwarfs to put an upper/lower limit to the mass of the object, and this may result a powerful tool for discriminating bona fide brown dwarfs of $M \leq 0.06 M_{\odot}$.

2. There is some pre-MS depletion in the solar model, but it can not reach the factor 100 required by the obsevational value of atmospheric ${ }^{7} \mathrm{Li}$ in the Sun, compared with the ${ }^{7} \mathrm{Li}$ of the solar nebula. Most recent models (Pinsonneault et al.1992b, D'Antona and Mazzitelli, 1992) tend to give a somewhat larger solar pre-MS depletion than previously found, due to the complex update of interior physics and of the ${ }^{7} L i+p$ reaction rate. Within the present uncertainties, we can conservatively say that ${ }^{7} L i_{\text {present } \odot} \sim(0.1-0.5)^{7} L i_{\text {initial }}$.

3. As discussed in the introduction, theoretical modeling seems to establish that there is one dominant mechanism for depletion among the pre-MS stars, and it is nuclear burning only. The earlier the evolutionary stage at which we compare model results and the observations, the more probable it is that we are witnessing "standard" depletion.

4. Low metallicity stars have smaller pre-MS depletion and "standard" models consistently explain population II observations (Deliyannis et al., 1990) although there are a number of other possibilities to achieve reasonable agreement with the data, which imply "long term" depletion mechanisms (Vauclair, 1988, Pinsonneault et al., 1992a).

There are a few other results which are important for their consequences:

1. No models of pre-MS, in the present context, can predict ${ }^{7} \mathrm{Li}$-burning at $L \gtrsim L_{\odot}$.

2. Observationally, we do expect a ${ }^{7} \mathrm{Li}$-depletion boundary, whose precise location is subject to the above discussed uncertainties.

3. ${ }^{7} \mathrm{Li}$ in luminous $\mathrm{T}$ Tauri must be the initial ${ }^{7} \mathrm{~L} i$ of the forming interstellar cloud. If we find $[\mathrm{Li} / \mathrm{H}]_{\text {today }} Z[\mathrm{Li} / \mathrm{H}]_{\text {solarsystem }}$, we get important information on the galactic evolution of ${ }^{7} L i$ in the latest 4.6 billion years.

Typical Li-depletion boundaries from pre-MS models are shown in figure 

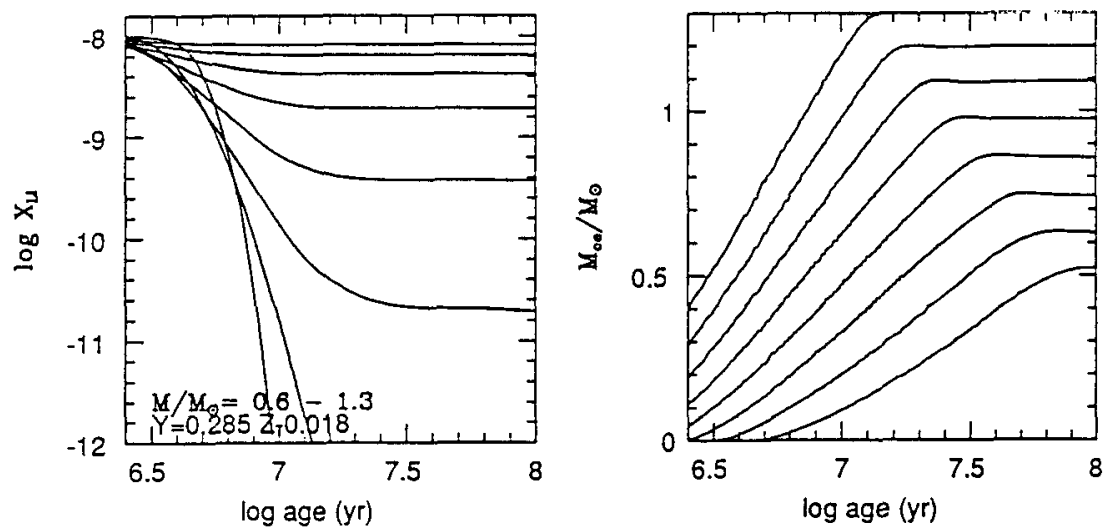

FIGURE VI Lithium abundances (left) and convective masses (right) versus age for models of standard pre-MS depletion with updated physics (D'Antona and Mazzitelli, 1992). The masses are increased by $0.1 M_{\odot}$ from 0.6 to 1.3 .

I. Until recently, observations have seemingly implied larger abundances of ${ }^{7} \mathrm{Li}$ in TTauri's than in young clusters (Strom et al. 1989a, Basri et al., 1991). Recent work by Magazzù et al., 1992 -see also the updated summary given in a poster paper by Martin et al.(1992)- revise the picture to give a remarkably constant $\log N_{L i} \sim 3.2 \pm 0.3$, complemented by remarkable depletion only in low luminosity TTs. Although the theoretical depletion boundaries and the location of depleted stars do not match perfectly each other, this should not worry us, in view of the wide discussed uncertainties in the location of both tracks and observational data in the HR diagram. The possible conclusion from Magazzù et al. data is that in the last few billion years from the time of formation of the solar system there has been low or null increase in the ${ }^{7} \mathrm{Li}$ content. If this is confirmed, it would dismiss the models of galactic evolution in which a considerable fraction of the present ${ }^{7} \mathrm{Li}$ comes out from a population of slowly evolving stars, which would contribute with an efficient production of ${ }^{7} L i$ from the time of the solar system to today (D'Antona and Matteucci, 1992).

Both Basri et al.(1991) and Magazzù et al.(1992) find no significant difference in ${ }^{7} L i$ abundance among CTTs and WTTs, or within CTTs having very different inferred $\dot{M}$ 's. Some TTs with very large $\dot{M}$ 's have very low ${ }^{7} L i$. While the first point seems to indicate that probably accretion has no strong influence on the atmospheric ${ }^{7} \mathrm{Li}$, the second one indicates that accretion is efficient also at stages in which ${ }^{7} L i$ is already depleted, at least in a few stars, and/or for a very short time-see section 3 . The effect of accretion on the final ${ }^{7} L i$ abundance should be investigated by numerical models. Let us suppose that a $0.8 M_{\odot}$ can accrete for $10^{6} \mathrm{yr}$ at a rate of $\sim 10^{-7} M_{\odot} / \mathrm{yr}$ and age log $\mathrm{t}=7.2$. Let us take, as in figure VI, the initial abundance of ${ }^{7} \mathrm{Li} X_{7 \mathrm{Li}} \sim 10^{-8}$ in mass fraction. The same figure shows that, at the given age, the initial value is already reduced to $X_{7 L i} \sim 5 \times 10^{-11}$, and that the convective envelope covers $\sim 0.3 M_{\odot}$. The star will become a $0.9 M_{\odot}$ with surface abundance $\sim 2.5 \times 10^{-9}$, while its abundance in the absence of mass accretion would have been $\sim 4 \times 10^{-10}$. This case is 
probably extreme, but it is clear that late accretion, which we have seen has no considerable influence on the pre-MS structural evolution, may influence the final ${ }^{7} \mathrm{Li}$ abundance at the surface, at least in the sense of providing a spread in the resulting abundance in the young MS stars. This possibility would be very unfortunate, as the spread, in this case, can not be attributed uniquely to the advection of other depletion mechanisms, acting, during the MS phase, in different degrees from star to star.

\section{REFERENCES}

Adams, F., C., Lada, C. J., Shu, F. H. 1987, ApJ 312, 788.

Alexander, D. R., Augason, G. C., Johnson, H. R. 1989, ApJ 345, 1014.

Barsony, M., Burton, M. G., Russell, A. P. G., Carlstrom, J. E., Garden, R. 1989, ApJ 346, L 93.

Basri, G. Bertout, C. 1989, ApJ 341, 340.

Basri, G., Martin, E. L., Bertout, C. 1991, $A \mathscr{E} A$ 252, 625.

Bertout, C. 1989, ARAEA 27, 351.

Bertout, C., Basri, G., Bouvier, J. 1988, ApJ 330, 350.

Bodenheimer, P. 1965, ApJ142, 451.

Bodenheimer, P. 1991, in Angular Momentum evolution of Young Stars, Kluwer, eds. S. Catalano and J. R. Stauffer, p. 1.

Boesgaard, A. M., Steigman, G. 1985, ARAEA 23, 319.

Bouvier, J. 1991, in Angular Momentum evolution of Young Stars, Kluwer, eds.

S. Catalano and J. R. Stauffer, p. 41.

Böhm Vitense, E. 1958, Zs. für Ap. 46, 108.

Bruston, P., Audouze, J., Vidal-Majar, A., Laurent, C. 1981, ApJ 243, 161.

Canuto, V. M., Mazzitelli, I. 1990, ApJ 370, 295 (CM).

Canuto, V. M., Mazzitelli, I. 1992, ApJ 389, 724.

Cox, A. N., Tabor, J. E. 1976, ApJS 31, 271.

D'Antona, F., 1991, in Evolutionary Phenomena in the Universe, ed. Compositori, Bologna, SIF Conference Proceedings, 32, 43.

D'Antoná, F., Matteucci, F. 1990 A $\mathcal{E} A$ 248, 62.

D'Antona, F., Mazzitelli, I. $1984 A \& A$ 138, 431.

D'Antona, F., Mazzitelli, I. 1985 ApJ 296, 502.

D'Antona, F., Mazzitelli, I. 1992, in preparation.

D'Antona, F., Mazzitelli, I., Gratton, R. 1992, $A \& A, 257,539$.

Däppen, W. 1992, in these Proceedings.

Deliyannis, C. P., Demarque, P. 1991, ApJ 379, 216.

Deliyannis, C. P., Demarque, P., Kawaler, S. D. 1990, ApJS 73, 21. 
Deliyannis, C. P., Pinsonneault, M. H. 1990, ApJ 367, L67.

Demarque, P. 1991, in Evolution of Stars: The Photospheric Abundance Connection, eds. G. Michaud and A. Tutukov, Kluwer, Dordrecht, p. 71.

Endal, A. S., Sofia, S. 1981, ApJ 243, 625.

Garcia Lopez, R. J., Spruit, H.C. 1991, ApJ 377, 268.

Grossman, A. S., Graboske, H. C. Jr. 1973, ApJ 180, 195.

Graboske, H. C. Jr., Olnes, R. J., Grossman, A. S. 1975, ApJ 199, 255.

Hartmann, L. W., Jones, B. F., Stauffer, J. R., Kenyon, S. J. 1991, AJ 101, 1050.

Herbig, G. H., Vrba, F. J., Rydgren, A. E. 1986, $A J$ 91, 575.

Huebner, W. F., Merts, A. ., Magee, N. H., Argo, M. F. 1977, Los Alamos Scientific Laboratory Report, No LA-6760-M.

Kenyon, S. J., Hartmann, L. W. 1987, ApJ 323, 714.

Kenyon, S. J., Hartmann, L. W. 1990, ApJ 349, 197.

Kurucz, R. L. 1991, in "Stellar Atmospheres: Beyond the Classical Models", L. Crivellari, I. Hubeny, D. G. Hummer eds., NATO ASI Series, Kluwer, Dordrecht.

Larson, R. B. 1969, MNRAS 145, 271.

Lynden-Bell, D., Pringle, J. E. 1974, MNRAS 168,603.

Magazzú, A., Rebolo, R., Pavlenko, Ya. V. 1992, ApJ, in press.

Magni, G., Mazzitelli, I. 1979, $A \& A$ 72, 134.

Martin, E. L., Garcia Lopez, R. J., Rebolo, R., Magazzú, A., Pavlenko, Ya. V. 1992 , in these proceedings.

Mathews, G. J., Alcock, C. R., Fuller, G. M. 1990 ApJ 349, 449.

Mazzitelli, I. 1989, in Low Mass Star Formation and Pre-Main Sequence Objects, e. B. Reipurth. ESO Conference Proceedings N. 33, 433.

Mihalas, D., Däppen, W., Hummer, D. G. 1988, ApJ 331, 815.

Mercer-Smith, J. A., Cameron, A. G. W., Epstein, R.I. 1984, ApJ 279, 363.

Montmerle, T., André, P. 1989, in Low Mass Star Formation and Pre-Main Sequence Objects, B. Reipurth ed., ESO Proceedings N. 33, 407.

Palla, F: 1991, in IAU Symp. 147: Fragmentation of molecular Clouds and Star Formation, ed. E. Falgarone, Kluwer.

Palla, F., Parigi, G. 1992, in these Proceedings.

Palla, F., Stahler, S. W. 1990, ApJ 360, L47.

Palla, F., Stahler, S.7 W. 1991, ApJ 375, 288.

Palla, F., Stahler, S. W. 1992, ApJ in press.

Pinsonneault, M. H., Kawaler, S., Demarque, 1990, ApJS 74, 501.

Pinsonneault, M. H., Deliyannis, C. P., Hobbs, L. M., Thornburn, J. A. 1991, in Cool Stars Workshop, Tucson. 
Pinsonneault, M. H., Deliyannis, C. P., Demarque, 1992a, ApJS 78, 179.

Pinsonneault, M. H., Deliyannis, C. P., Demarque, 1992b, in these Proceedings.

Pozio, F. 1990, thesis, University of Rome, unpublished.

Pozio, F. 1991, Mem. Soc. Astr. It. 62, 171.

Pringle, J. E. 1989, in Low Mass Star Formation and Pre-Main Sequence Objects, e. B. Reipurth, ESO Conference Proceedings N. 33 p. 89.

Proffitt, C. R., Michaud, G. 1989, ApJ 346, 976.

Proffitt, C. R., Michaud, G., Richer, J. 1990, in "Cool Stars, Stellar Systems and the Sun, ed. G. Wallerstein, (ASP Conference Series 9), p. 35.

Rebolo, R., Martin, E. L., Magazzú, A. ApJ 389, L83.

Ryan, S. G., Norris, J. E., Bessell, M. S., Deliyannis, C.P. 1992, ApJ 388, 184.

Rieke, G. H., Ashok, N. M., Boyle, R. P. 1989, ApJ 339,

Rieke, G. H., Rieke, M. J., Morgan, W. T. 1991, in Astrophysics with Infrared Arrays, ed. R. Elston, ASP Conference Series, p. 244.

Rogers, F. J., Iglesias, C. A. 1992, ApJS 79 (RI).

Saumon, D. 1990, ph.D. Thesis, Rochester University.

Schatzman, E. 1991, in Angular Momentum evolution of Young Stars, Kluwer, eds. S. Catalano and J. R. Stauffer, p. 223.

Stahler, S. W. 1983, ApJ 274, 822.

Stahler, S. W. 1988, ApJ 332, 804.

Stahler, S. W., Shu, F. H., Taam, R. E. 1980, ApJ 241, 637.

Stahler, S. W., Palla, F. 1990, ApJ 360, L47.

Stevenson, D. J. 1991, ARA\&A 29, 163.

Strom, K. M., Strom, S. E., Edwards, Cabrit, Skrutskie 1989a, AJ 97, 1451.

Strom, K. M.,, Wilkin, F. P., Strom, S. E., Seamon, R. L. 1989 b, AJ 98, 1444.

Swenson, F. J., Stringfellow, G. S., Faulkner, J. 1990, ApJ 348, L33.

York, D. G., Rogerson, J. B. 1976, ApJ 203, 378.

Vandenberg, D. A., Poll, H. E. 1989, AJ 98, 1451.

Vauclair, S. 1988, ApJ 335, 971.

Vidal-Majar, A., Laurent, C., Gry, C., Bruston, P., Ferlet, R., York, D.G. 1983, $A \& A 120,58$.

Walter, F. M. 1987, PASP 99, 31.

Walter, F. M., Brown, A., Mathieu, R. D., Myers, P. C., Vrba, F. J. 1988, AJ 96, 297.

Zinnecker, H., McCaughrean, M. J., Wilking, B. A. 1992, in Protostars and Planets III, ed. E.H. Levy, J. I. Lunine, M.S. Mathews, (University of Arizona Press: Tucson, Arizona), in press. 